\title{
Liebe zum Detail
}

\author{
André Blondiau a, Sabine Rommerskirchen ${ }^{\mathrm{b}}$, Sarah Stölting ${ }^{\mathrm{c}}$, Henrik Pfahlerd, Lars Clarfeld ${ }^{\mathrm{e}}$ \\ ${ }^{a}$ Dr. oec. HSG, Medizincontroller, ${ }^{b}$ Medizincontrollerin, ${ }^{c}$ Medizincontrollerin, ${ }^{d}$ Dr. med. Leiter Medizinsteuerung, \\ e Dr. med., Leiter strategisches Medizincontrolling, Mitglied FMH \\ Alle Autoren arbeiten zum Zeitpunkt der Erstellung des Artikels im Strategischen Medizincontrolling (Ärztliche Direktion) der Insel Gruppe AG in Bern.
}

Im Kontext steigenden Kostendrucks und hoher Vorhalteleistungen stellt das Medizincontrolling als Bindeglied zwischen Management und Kliniken eine wichtige Rolle bei der gezielten Spitalentwicklung. Es unterstützt bei Prozessoptimierungen und Potentialidentifikation. Im folgenden Artikel werden das Integrierte Controlling-Konzept an der Insel-Gruppe AG vorgestellt und Verbesserungspotentiale skizziert.

Schweizer Spitäler sehen sich über die letzten Jahre mit wachsenden Herausforderungen, wie z.B. dem steigenden Kostendruck und zunehmenden Qualitätsansprüchen konfrontiert. [1]. Zu den Hauptursachen hierfür zählen die im Rahmen der neuen Spitalfinanzierung schrittweise sinkende Baserate und schrumpfende gemeinwirtschaftlichen Leistungen seitens der Kantone. Gleichzeitig sind die Verhandlungen mit den Kantonen und den Versicherern schwieriger geworden, was zu einem Missverhältnis zwischen angebotener Leistung und Vergütung führt. Spitäler sind daher gut beraten, Redundanzen im Leistungserbringungsprozess zu vermeiden, um weitere Kostensteigerungen zu verhindern [2-4].

\section{Der Druck für Universitätsspitäler steigt}

Kleinere Spitäler haben die Chance, auf diese Situation mit einer Spezialisierung ihres Leistungsportfolios zu reagieren [2, 5]. Für Zentrums- und Maximalversorger wie Universitätsspitäler sind die Herausforderungen durch diesen Paradigmenwechsel jedoch tiefgreifender. Aufgrund ihres breiteren Patientenportfolios und ihres Aus- und Weiterbildungsauftrages sind die not-

\section{Résumé}

Dans le contexte de la pression des coûts et de haute prélèvement, le contrôle médical joue un rôle important en tant que lien entre la direction et les cliniques dans le développement hospitalier ciblé. II favorise I'amélioration des processus et l'identification des potentiels. L'article suivant présente le concept de contrôle intégré à l'Hôpital universitaire de Berne et décrit le potentiel d'amélioration dans la pratique clinique. wendigen Vorhalteleistungen höher als bei anderen Spitälern. Dennoch rechnen auch sie über das pauschalierte Finanzierungssystem SwissDRG ab. Aus diesem Grund wird Universitätsspitälern aktuell noch eine höhere Baserate gewährt, doch dieser Finanzierungspuffer schrumpft kontinuierlich [6]. Universitätsspitäler kommen damit zunehmend unter Effizienzzwang und müssen sich mit kleineren Spitälern messen, die ihr Behandlungsportfolio einfacher spezialisieren können. Anders als bei den kleinen und spezialisierten Häusern spielt die Verbesserung der Zusammenarbeit zwischen unterschiedlichen Fachbereichen eine entscheidende Rolle für die Qualität und Effizienz integrierter, fachübergreifender Behandlungsprozesse [5, 7, 8]. In diesem Balanceakt zwischen Steigerung der Effizienz auf der einen Seite und Stabilisierung der medizinischen Leistungsqualität auf der anderen Seite ist es wichtig, die richtigen Entscheidungen auf der Basis verlässlicher Daten und Informationen zu treffen.

\section{Medizincontrolling als Brücke zwischen Kliniken und Gesamtführung}

In grösseren Häusern fällt diese Steuerungsrolle in die Verantwortung von Medizin- und Finanzcontrolling. Die Hauptherausforderung besteht darin, die Kliniken bei der Dokumentation und Abrechnung ihrer Leistungen zu unterstützen, Leistungs- und Kostendaten zu analysieren und gleichzeitig die Geschäftsleitung zu informieren, so dass eine nachhaltige Spitalführung möglich ist. Das Medizincontrolling analysiert dabei aus Austrittssicht und hat die Leistungen im Fokus, während das Finanzcontrolling über das Budget aus Sicht der Ergebniscenterperspektive steuert. Die enge 
Zusammenarbeit zwischen Finanz- und Medizincontrolling ist wichtig für eine hohe Informationsverlässlichkeit und daher Grundvoraussetzung, um ein aussagekräftiges Medizincontrolling-Reporting zu erstellen. Durch den gezielten Einsatz spezifischer IT-Tools und der kontinuierlichen Zusammenarbeit zwischen Führungskräften der Kliniken und Mitarbeitern von Medizin- und Finanzcontrolling werden die Kliniken gezielt dabei unterstützt, ihr Leistungsportfolio an den systembedingten Anforderungen zu orientieren und nachhaltig zu wirtschaften. Gleichzeitig können durch die enge und regelmässige Zusammenarbeit zwischen Klinik und den Controllingbereichen die Kliniken besser an der Gesamtstrategie des Hauses ausgerichtet werden.

\section{Integriertes Controlling - Strategisches Steuerungsinstrument an der Insel Gruppe AG}

Das Medizincontrolling gilt als Schnittstelle zwischen Medizin und Management im Spital und hat neben Kodierung und DRG-Systemweiterentwicklung die Aufgabe, die stationären Leistungsdaten unter ökonomischen Aspekten zu analysieren. Am Universitätsspital Bern wurden daher bis 2015 viermal pro Jahr stationäre Leistungskennzahlen aller Kliniken im Rahmen eines Quartalsreporting durch das Medizincontrolling analysiert und in der Folge nötige Massnahmen lanciert (Abbildung 1 skizziert schematisch den Ablauf des Medizincontrolling Klinikreportings). Der Analyseprozess beginnt auf der Klinikebene. In der Folge wird das DRG-Portfolio analysiert, um schliesslich auffällige Fälle genau unter die Lupe zu nehmen.

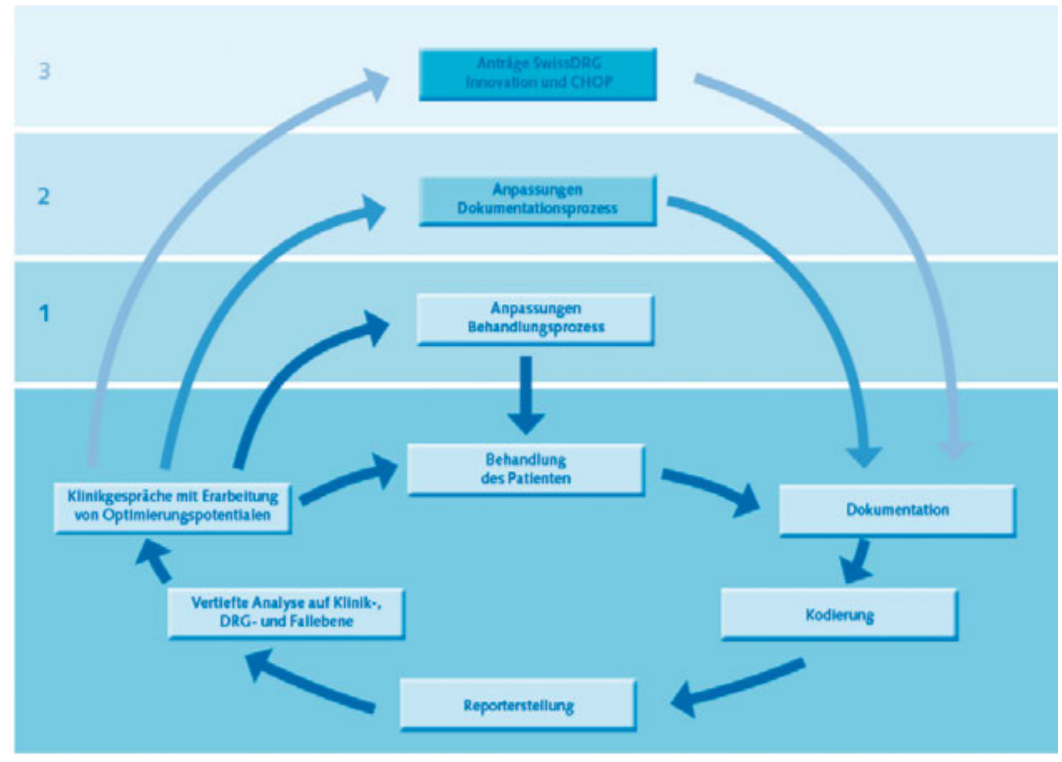

Abbildung 1: Der Managementkreislauf des quartalsweise durchgeführten MedCoKlinikreportings am Inselspital.
Im Rahmen des Zusammenschlusses zwischen Inselspital und Spitalnetz Bern zur Insel Gruppe AG wurde der Klinikreportingprozess angepasst und verdichtet. Im Rahmen der «Integrierten Controllingsitzung» treffen sich Vertreter des administrativen Supportbereichs der Kliniken sowie des Finanz- und des Medizincontrollings und diskutieren vierteljährlich die Leistungszahlen aller Kliniken. Aufgrund der Entwicklung wichtiger Leistungskennzahlen wie zum Beispiel dem Ergebnis, der Fallzahl, des Case Mix oder des Case Mix Index, wird in der Sitzung entschieden, ob vertiefende Gespräche in Kliniken mit auffälliger Leistungsentwicklung stattfinden sollten.

Der Prozess wurde aus zwei Gründen adaptiert: Zum einen wurden zur Verbesserung der Konsistenz die Frequenz und die Inhalte der Reportings zwischen den beiden Controllingbereichen synchronisiert. Zum anderen konnte der Prozess über die Einführung von Standards effizienter gestaltet werden. Wurden die Kliniken früher sowohl aus finanz- als auch aus medizincontrollerischer Sicht unabhängig beurteilt, so steht heute das Gesamtbild als gemeinschaftliche Beurteilung durch beide Bereiche im Mittelpunkt. Des Weiteren werden Klinikreportinggespräche nicht mehr standardmässig durchgeführt, sondern nur bei Bedarf, wenn sich wichtige Leistungszahlen auffällig verändert haben.

\section{Primäranalyse wichtiger Basiskennzahlen}

Im ersten Analyseschritt geht es darum, einen Überblick über die Situation der Klinik zu gewinnen und wichtige Trends zu identifizieren. Die Leistungszahlen werden mit denen des Vergleichszeitraums des Vorjahres verglichen, um Veränderungen identifizieren und von saisonalen Schwankungen unterscheiden zu können. Zu diesen Kennzahlen gehört zum Beispiel die Entwicklung der Fallzahl als genereller Indikator. Darüber hinaus werden relationale Kennzahlen wie Day Mix Index ${ }^{1}$, Kosten pro Case-Mix-Punkt ${ }^{2}$ sowie der Verweildauerquotient ${ }^{3}$ als wichtige Indikatoren für die Effizienz beurteilt.

Um Aussagen über zeitliche Zusammenhänge bestimmter Auffälligkeiten machen zu können, werden die wichtigsten Kennzahlen zusätzlich im zeitlichen Verlauf analysiert (Abbildung 2). Die Analyse dieser Standardparameter ermöglicht einen guten Überblick über den Status Quo und die Entwicklungen im Vergleich zum Vorjahr der Klinik. Um Schwankungen zwischen den Betrachtungsperioden erklären zu können, werden in einem nächsten Schritt detaillierter das DRG-Portfolio, sowie einzelne Extremfälle analysiert. 

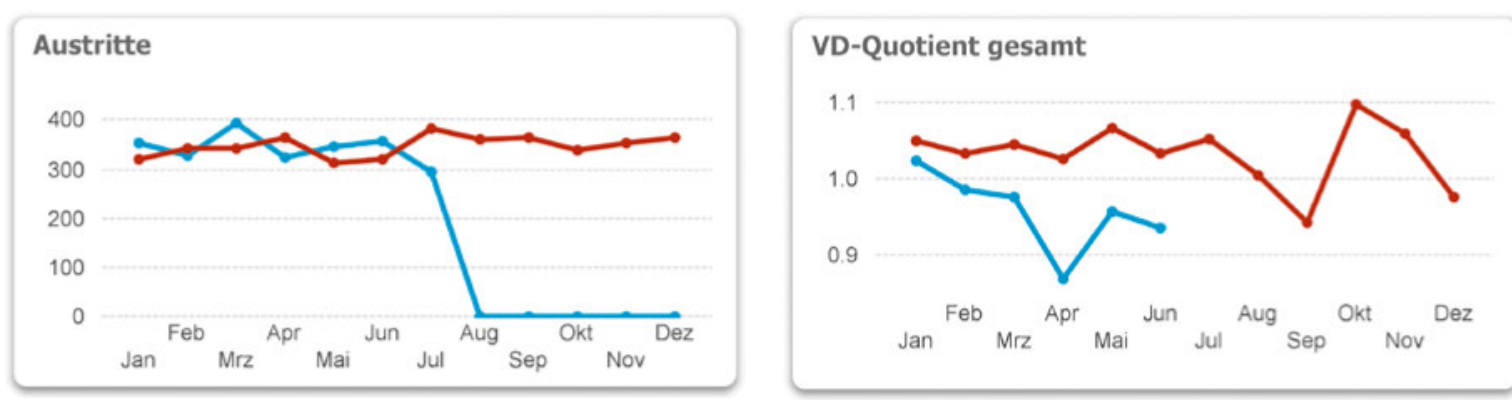

Abbildung 2: Ausschnitt aus der Verlaufsanalyse des MedCo-Klinikreportings für die Primäranalyse.

\section{Auf die Details kommt es an}

Während die bisher dargestellte Analyse zumeist beschreibender Natur ist, haben die weiteren Analysen zunehmend erklärenden Charakter. Bei der Aufarbeitung werden unter den häufigsten Fallpauschalen die verlustreichsten DRG genauer auf den Anteil Verlustfälle, auf den Verweildauerquotient sowie auf Kostendeckungsgrad und -verteilung hin analysiert. Die detaillierte Analyse erfolgt über die Betrachtung der Kostenträgerrechnung und des Kosten-Erlösverhältnisses (Abbildung 3). Die Kosten werden dabei den Erlösen einer bestimmten DRG in Abhängigkeit ihrer Verweildauer gegenübergestellt. Liegen die Kosten der Fälle oberhalb der Erlöskurve, sind die Fallkosten höher als der zu erwartende Erlös und der Fall generiert ein Defizit. Im Umkehrschluss generiert ein Fall einen Gewinn, wenn die Kosten unterhalb der Erlöskurve liegen. Eine Häufung von Fällen, die bei ähnlicher Liegezeit deutlich über der Erlöskurve liegen, deutet auf hohe Kosten innerhalb dieser DRG hin. Da die fallzahlstärksten DRGs das Gesamtergebnis der Klinik entscheidend beeinflussen, bildet ihre genaue Analyse

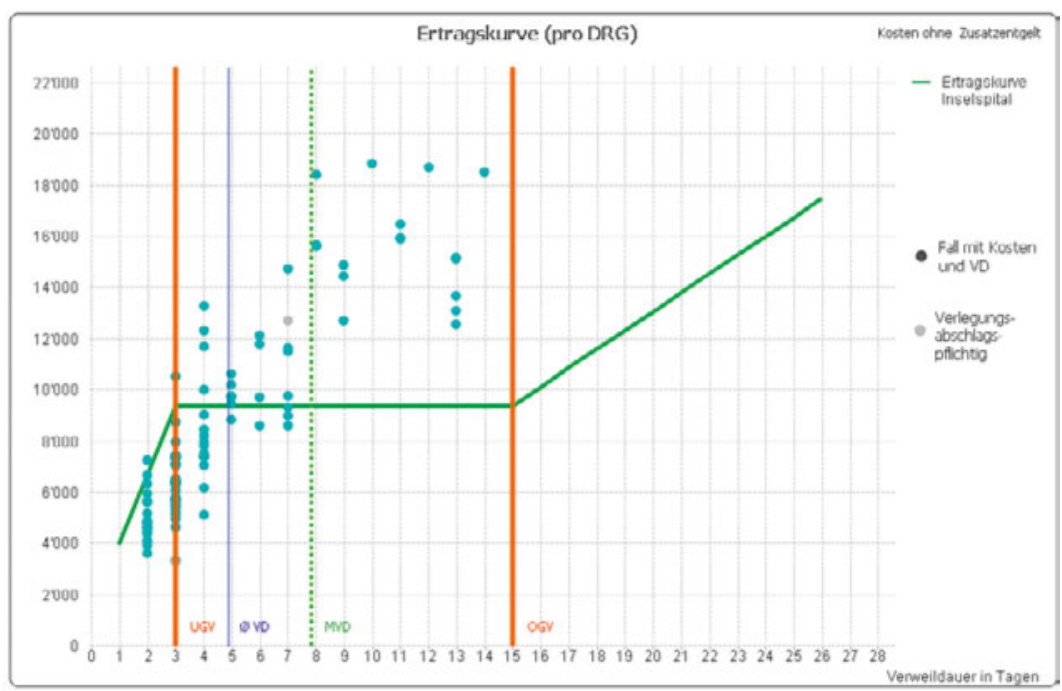

Abbildung 3: Die Ertragskurve stellt die Kosten der Fälle einer bestimmten DRG im Verhältnis zu der spezifischen Vergütung dar. (UGV = untere Grenzverweildauer; MVD = Mittlere Verweildauer; OGV = obere Grenzverweildauer; VD = Verweildauer) die Grundlage für Verbesserungsmöglichkeiten auf prozessualer (z.B. Aufenthaltsdauer) oder systemischer (DRG-Antrag) Ebene und ist damit entscheidender Stellhebel für die Klinikleitung.

Der abschliessende Analyseschritt findet auf der Fallebene statt. Auf Basis der Falllisten einer Klinik wird nach extrem defizitären oder anderweitig auffälligen Fällen, sogenannten Extremfällen, gesucht und diese werden vertieft analysiert. In diesem Arbeitsschritt können zum Beispiel Fehl-Erfassungen und Kostentreiber identifiziert und korrigiert werden.

\section{Interventionswege}

Die Ergebnisse der zuvor aufgearbeiteten Analysen werden mit den Klinikern ${ }^{4}$ interprofessionell diskutiert und potentiell nötige Veränderungen initiiert. Je nach Bedarf werden in der Konsequenz entweder der Behandlungsprozess bzw. die Prozessschritte überarbeitet und/oder der Leistungserfassungsprozess angepasst. Sind die Änderungsanforderungen systemischer Natur, können über Werkzeuge wie Innovations-, SwissDRG- oder CHOP-Anträge Anpassungen initiiert werden (Abbildungen 1 und 4). Diese Massnahmen werden durch Mitarbeiter aus dem Medizincontrolling gesteuert. Im Folgenden werden die unterschiedlichen Interventionswege genauer beschrieben.

\section{Anpassungen im Behandlungsprozess und in der Dokumentation}

Ergeben sich aus den Analysen grössere Unklarheiten oder Redundanzen bei bestimmten Behandlungsprozessen, werden die betroffenen Prozesse oder Prozessschritte genauer untersucht. In diesen Prozessanalysen arbeiten Mitarbeiter der Klinik eng mit dem Medizincontrolling und dem Finanzcontrolling zusammen. Die Federführung liegt dabei bei der Klinik. Durch die situative Ergänzung von Kompetenz durch Mitarbeiter verschiedener Abteilungen werden Veränderungsinitiativen schnell und zielgerichtet abgeleitet. Beispiel hierfür ist die bereichsübergreifende Zusammenarbeit im Pro- 


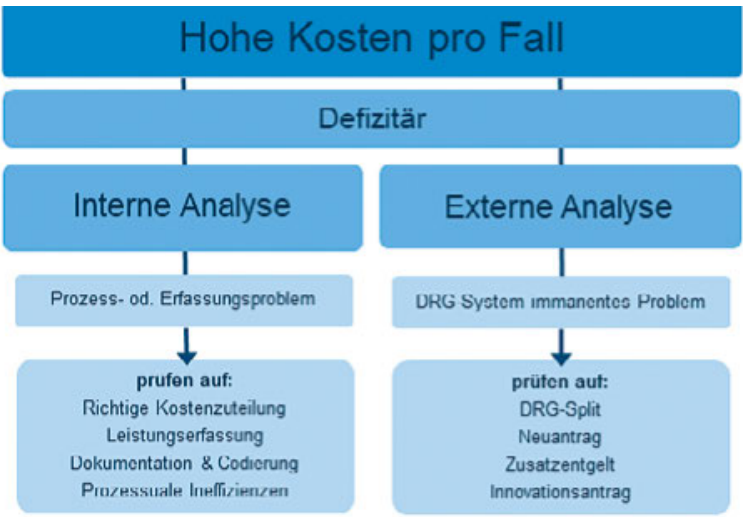

Abbildung 4: Analyseschema des strategischen Medizincontrollings zur Identifikation und Bearbeitung von hochdefizitären Fällen

zess der Rehabilitationsfolgebehandlung, um unnötige Wartezeiten für den Patienten zu reduzieren. Neben dem Behandlungsprozess wird auch die Dokumentation analysiert, da ein häufiger Grund für Unterdeckung in einer unzureichenden Dokumentation und Erfassung liegt. Bei der Vermutung auf Dokumentationsmängel werden die Mitarbeiter wiederholt geschult und/oder der Dokumentationsreferenzprozess in der Klinik angepasst.

\section{Innovations- und SwissDRG-Anträge}

Ergeben die Analysen und Gespräche, dass spezifische DRGs oder Behandlungen nicht richtig abgegolten sind, werden diese DRGs im Medizincontrolling genauer analysiert. Stellt sich dabei heraus, dass ein Eingriff nicht oder nur unzureichend im System abgebildet ist, werden Anträge bei der SwissDRG AG zur Anpassung bestimmter Fallpauschalen oder beim Bundesamt für Statistik zur Anpassung des Operationskatalogs gestellt (vgl. Abbildung 1, Weg 3). Dieser Prozess wird durch die Mitarbeiter des Medizincontrollings lanciert und gemeinsam mit den Kliniken ausgearbeitet. Darüber hinaus besteht im Kanton Bern die Möglichkeit, über Innovationsfinanzierung neue und bis dato unzureichend finanzierte Verfahren finanziell

\section{Grundlage für nachhaltiges und kooperatives Krankenhausmanagement}

Ob nun kleine, lokale Prozessverbesserungen, wie die Verbesserung der Zusammenarbeit zwischen verschiedenen Abteilungen im Beispiel der Rehabilitationsorganisation, oder weitreichende Anpassungen in der Finanzierungssystematik: das gut funktionierende Zusammenspiel zwischen den Kliniken und den verschiedenen Controllingbereichen erlaubt einen kontinuierlichen Wissensaustausch zwischen den medizinischen und den administrativen Bereichen. Es ist also eine wichtige Voraussetzung für eine kontinuierliche Verbesserung des medizinischen Kerngeschäfts an der Insel Gruppe AG.

Das Medizincontrolling als Bindeglied zwischen diesen Ebenen unterstützt in seiner begleitenden, orchestrierten Funktion die Kliniken bei der Erfüllung ihrer medizinischen und ökonomischen Ziele, indem Qualitäts- und Erlöspotentiale identifiziert, sinnvoller Ressourceneinsatz ermöglicht und Veränderungsinitiativen initiiert und koordiniert werden. In dieser Unterstützungsfunktion entlastet das Medizincontrolling die Kliniken und ermöglicht fundierte Entscheidungen durch die Geschäftsführung. In Zeiten hohen Kostendrucks und zunehmender Entscheidungskomplexität bildet dieses enge Zusammenspiel zwischen den Kliniken und den administrativ unterstützenden Bereichen eine wichtige Grundlage für fundierte, nachhaltige Entscheidungen. In dieser Rolle kann das Medizincontrolling als wertvolle Brücke zwischen dem medizinischen Kernbereich und den administrativ steuernden Einheiten verstanden werden und bildet ein zentrales Fundament für ein modernes, zielgerichtetes Krankenhausmanagement.

\section{Referenzen}

1 Kocher G. Gesundheit und Gesundheitssysteme in Europa - ein aktueller Ländervergleich. Schweiz Ärztezeitung, 2013;9: p. 345-7.

2 Gemmel P, Vandaele D, and Tambeur W. Hospital Process Orientation (HPO): The development of a measurement tool. Total Quality Management \& Business Excellence, 2008;19(12) p. 1207-17.

3 Kohlbacher M, Gruenwald S. Process orientation: conceptualization and measurement. Business Process Management Journal, 2011;17(2): p. 267-83.

4 Mehra T, Bitzi C. SwissDRG - von Fallkosten zu Kostengewichten Schweizer Ärztezeitung, 2014;95(50): p. 1917-9.

5 Vos L, et al. Towards an organisation-wide process-oriented organisation of care: A literature review. Implementation science: IS, 2011;6(1): p. 1-14.

6 Kopf R, et al. Eine vergleichende Analyse der Basisfallwerte in der Schweiz und in Deutschland. Schweizer Ärztezeitung, 2014;44: p. 6

7 Blondiau A. Prozessorientierung im Krankenhaus - Voraussetzungen und Erfolgsfaktoren für eine erfolgreiche Umsetzung, in Handbuch für Integrierte Versorgung - Strategien, Konzepte, Praxis, W. Hellmann, Editor. 2012, medhochzwei Verlag Heidelberg.

8 Rüegg-Stürm J. et al. Management komplexer Wertschöpfungsprozesse im Gesundheitswesen: Vernetzung beginnt in der Organisation, in Vernetzung im Gesundheitswesen: Wettbewerb und Kooperation, V.E. Amelung, J. Sydow, and A. Windeler, Editors. 2008, W. Kohlhammer Verlag: Stuttgart. p. 420. 